\title{
A Fast Algorithm for Single Image Dehazing Based on Estimating Illumination Veil
}

\author{
Xumeng Chen \\ ICIE Institute, School of Aerospace Science and \\ Technology, Xidian University, \\ Xi'an, China \\ e-mail: xmengchen@126.com
}

\begin{abstract}
We propose a new approach based on estimating illumination veil. The algorithm for non-uniform illumination of fog image has a good result. Given a degraded image, minimum intensity of RGB components of each point can be figured out as haze energy veil with the proposed guided filter, subtracting the hazy energy veil form the hazy image, then maximum intensity of residual RGB components of each point can calculated and filtered as the scene illumination veil. Consequently, the RGB reflectance of each point can be obtained from the illumination/reflection imaging model. The proposed approach can dehaze images and enhance contrast simultaneously, and the global atmospheric light does not need to be calculated to avoid the risk of estimation errors. The proposed algorithm can also be applied to eliminating the scene illumination. Experiment results on a variety of outdoor hazy images demonstrate that the proposed method achieves good restoration in terms of visibility and color fidelity.
\end{abstract}

Keywords-Single image dehazing; dark channel prior; scene illumination; illumination veil; guided filter

\section{I . INTRODUCTION}

In bad weather conditions, the outdoor scene of color and contrast will usually be affected. The big particle medium of fog haze, soot will reduce the atmosphere veil brightness and illumination veil brightness which leads to some of the characteristics of visibility of an object greatly reduced, seriously affects the analysis of image characteristics, navigation and tracking of the human eye, outdoor monitor system, computer vision system, automatic navigation system and so on. In recent years, with the rapid development of computer hardware and software technology, the image dehazing algorithm[1, 2, 3] has made a great progress .He et al. [4]proposed a method based on dark channel prior and the atmospheric veil is estimated using dark channel prior and refined by soft matting. Original algorithm estimated the atmosphere light as a global constant by dark channel prior, calculated the atmospheric transmission by atmospheric scattering model and optimize transmission.. He et al.'s algorithm has been adopted and improved by many researchers $[5,6]$, but there are still some problems remaining to be addressed. Global atmospheric light is a very important parameter. The dehazing algorithm based on dark channel prior estimate the atmosphere light which is not enough

\author{
Wei Sun* \\ ICIE Institute, School of Aerospace Science and \\ Technology, Xidian University, \\ Xi'an, China \\ *Corresponding author: wsun@xidian.edu.cn
}

accurate to influence the dehazing results due to the unknown image depths.

According to atmosphere scattering model, this paper combines the atmosphere light and transmission of expression defined as the illumination veil. The value of atmospheric light will no longer be estimated which improves the operation speed and accuracy, reduces the operation time.

\section{THE AMOSPHERE SCATERING MODEL}

In computer vision and computer graphics, the atmospheric scattering model proposed by Koschmieder in fog, haze weather conditions, Fig .1, is widely used in dehazing technology research.

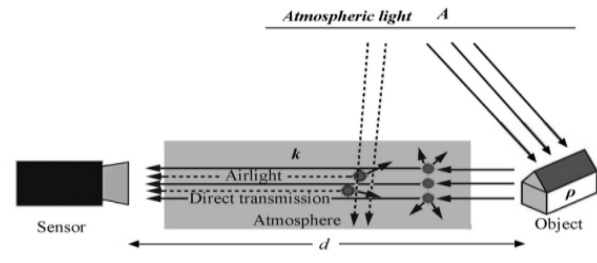

Figure 1. Atmospheric scattering model

The model described as follow:

(1)

$$
\mathrm{L}(\mathrm{x}, \mathrm{y})=\mathrm{L}_{0}(\mathrm{x}, \mathrm{y}) \mathrm{e}^{-\mathrm{kd}(\mathrm{x}, \mathrm{y})}+\mathrm{A}\left(1-\mathrm{e}^{-\mathrm{kd}(\mathrm{x}, \mathrm{y})}\right)
$$

where $\mathrm{L}(\mathrm{x}, \mathrm{y})$ is the observed hazy image, $\mathrm{L}_{0}(\mathrm{x}, \mathrm{y})$ is the clear haze-free image, $\mathrm{d}(\mathrm{x}, \mathrm{y})$ is the distance of corresponding object, $\mathrm{A}$ is the constant defined as global atmospheric light value, and $\mathrm{k}$ is the scatter coefficient of the atmosphere.

\section{ALGORITHM PROPOSED}

\section{A. Algorithm Inference}

According to Lambert's law, the illumination reflectance model claims that the intensity of any pixel is the product of the illumination of the scene and the reflectance of the objects in the scene, no matter where you look from for a diffusely reflecting surface. According to Eq. (1), $\mathrm{L}_{0}(\mathrm{x}, \mathrm{y})$ can be written as:

$$
\mathrm{L}_{0}(\mathrm{x}, \mathrm{y})=\mathrm{I}_{0}(\mathrm{x}, \mathrm{y}) \rho(\mathrm{x}, \mathrm{y})
$$

Where $I_{0}(x, y)$ is defined as the illumination veil function and $\rho(x, y)$ arises from the properties of the 
scene objects themselves. According to Eq.1 and Eq.2, the atmospheric scattering model can be changed as follow:

$\mathrm{L}(\mathrm{x}, \mathrm{y})=\mathrm{I}_{0}(\mathrm{x}, \mathrm{y}) \rho(\mathrm{x}, \mathrm{y}) \mathrm{e}^{-\mathrm{kd}(\mathrm{x}, \mathrm{y})}+\mathrm{A}\left(1-\mathrm{e}^{-\mathrm{kd}(\mathrm{x}, \mathrm{y})}\right)$

According to Eq. (3), we combine the atmosphere light and transmission of expression defined as the illumination veil. As follow:

$$
\mathrm{V}(\mathrm{x}, \mathrm{y})=\mathrm{A}\left(1-\mathrm{e}^{-\mathrm{kd}(\mathrm{x}, \mathrm{y})}\right)
$$

Therefore Eq. (3) can be rewritten as:

$$
\mathrm{L}(\mathrm{x}, \mathrm{y})=\mathrm{L}_{0}(\mathrm{x}, \mathrm{y}) \mathrm{e}^{-\mathrm{kd}(\mathrm{x}, \mathrm{y})}+\mathrm{V}(\mathrm{x}, \mathrm{y})
$$

According to Eq. (5), we can calculate the residual image $\mathrm{L}_{\mathrm{s}}(\mathrm{x}, \mathrm{y})$, we have:

$$
\mathrm{L}_{\mathrm{s}}(\mathrm{x}, \mathrm{y})=\mathrm{L}_{0}(\mathrm{x}, \mathrm{y}) \mathrm{e}^{-\mathrm{kd}(\mathrm{x}, \mathrm{y})}=\mathrm{L}(\mathrm{x}, \mathrm{y})-\mathrm{V}(\mathrm{x}, \mathrm{y})
$$

Put Eq.(2) into Eq.(6), we have:

$$
\mathrm{L}_{\mathrm{s}}(\mathrm{x}, \mathrm{y})=\mathrm{I}_{0}(\mathrm{x}, \mathrm{y}) \rho(\mathrm{x}, \mathrm{y}) \mathrm{e}^{-\mathrm{kd}(\mathrm{x}, \mathrm{y})}
$$

In this paper, we take $\mathrm{I}(\mathrm{x}, \mathrm{y})$ as the illumination of the scene, it can be rewritten as:

$$
\mathrm{I}(\mathrm{x}, \mathrm{y})=\mathrm{I}_{0}(\mathrm{x}, \mathrm{y}) \mathrm{e}^{-\mathrm{kd}(\mathrm{x}, \mathrm{y})}
$$

Put Eq.(8) into Eq.(7), we have:

$$
\rho(x, y)=L_{s}(x, y) / I(x, y)
$$

where $\rho(x, y)$ is the reflectance, which is the hazing-free image.

According to the above inference, in order to get the reflectance, we just need to estimate the atmospheric veil $\mathrm{V}(\mathrm{x}, \mathrm{y})$, residual image $\mathrm{L}_{\mathrm{s}}(\mathrm{x}, \mathrm{y})$ and illumination veil $\mathrm{I}(\mathrm{x}, \mathrm{y})$.

\section{B. Atmospheric Veil Estimation}

In common algorithm, researchers mainly estimate the atmospheric light A by looking for the brightest point in dark channel. However, because some brightest[7] pixels in white objects in the scene maybe brighter than global atmospheric light, which leads to undesirable selection of the global atmospheric light. The algorithm we proposed avoids the risk of the atmospheric light A estimation, which increases the accuracy of the operation. According to above inference, put Eq.(4) into Eq.(3), we have:

$$
V(x, y)=L(x, y)-I_{0}(x, y) \rho(x, y) e^{-k d(x, y)}
$$

According to Eq.(10), when given $\mathrm{d}(\mathrm{x}, \mathrm{y}) \rightarrow \infty$ and $\rho(x, y) \rightarrow 0$, we could estimate the atmospheric veil $V(x, y)=\lim _{\rho(x, y) \rightarrow 0} L(x, y)$.As a matter of fact, $\mathrm{d}(\mathrm{x}, \mathrm{y}) \rightarrow \infty$ can not be guaranteed in every image. However, for a small patch of color image, there must be at least one of $\mathrm{R}, \mathrm{G}$ or $\mathrm{B}$ components that are close to the zero reflection, whose reflectance coefficient approximately equals 0.In this paper, we estimate the atmospheric veil $\mathrm{V}(\mathrm{x}, \mathrm{y})$ by He's dark channel. As follows:

$$
\mathrm{V}_{\mathrm{dc}}(\mathrm{x}, \mathrm{y})=\min _{\mathrm{c} \in\{\mathrm{R}, \mathrm{G}, \mathrm{B}\}} \mathrm{L}_{\mathrm{c}}(\mathrm{x}, \mathrm{y})
$$

The value of the atmospheric veil $V(x, y)$ is a key parameter. We must adopt a reliable technology for optimization of the atmospheric veil. The guided filter $[8,9,10]$ performs fast edge-preserving smoothing property, which make the guided filter to be the best choice. Its principle is to put a good characteristics image as guidance image. Output image keeping the original features of the input image gets the edge of the guidance image detail features. The output image $q$ is linear correlation about the guidance image I and the input image p. Mathematical expressions are as follows:

$$
\begin{gathered}
\mathrm{q}_{\mathrm{i}}=\sum_{\mathrm{j}} \mathrm{W}_{\mathrm{ij}}(\mathrm{I}) \mathrm{p}_{\mathrm{i}} \\
\mathrm{W}_{\mathrm{ij}}(\mathrm{I})=\frac{1}{|\mathrm{w}|^{2}} \sum_{\mathrm{k}:(\mathrm{i}, \mathrm{j}) \in \mathrm{w}_{\mathrm{k}}}\left(1+\frac{\left(\mathrm{I}_{\mathrm{i}}-\mu_{\mathrm{k}}\right)\left(\mathrm{I}_{\mathrm{j}}-\mu_{\mathrm{k}}\right)}{\sigma_{\mathrm{k}}^{2}+\epsilon}\right)
\end{gathered}
$$

Where $\mathrm{W}_{\mathrm{k}}$ is a square window of a radius $\mathrm{r}, \mu_{\mathrm{k}}$ and $\sigma_{\mathrm{k}}^{2}$ are the mean and variance of $\mathrm{I}$ in $\mathrm{W}_{\mathrm{k}}, \epsilon$ is a regularization parameter, $|\mathrm{w}|$ is the number of pixels in $\mathrm{W}_{\mathrm{k}}$. we should choose appropriate the value of $\mathrm{W}_{\mathrm{k}}$ and $\epsilon$ for image.

Here, the guidance image $I$ is the image $\mathrm{V}_{\mathrm{dc}}(\mathrm{x}, \mathrm{y})($ Fig .2 (b) ) keeping the edge detail features by He's dark channel algorithm. Then, we must choose the input image $\mathrm{p}$ by gray opening of dark channel. We put the new image $V^{\prime}(x, y)$ as the input image. Let $V_{d c}(x, y)$, $\mathrm{V}^{\prime}(\mathrm{x}, \mathrm{y})$ input through guided filter processing. The result after filtering is the atmospheric veil $\mathrm{V}(\mathrm{x}, \mathrm{y})$ (Fig .2 (d) ).

\section{Illumination Veil Estimation}

The estimation of illumination veil is very important, which directly affects to the quality of the dehazing. According Eq.(9), We need to estimate the residual image $\mathrm{L}_{\mathrm{s}}(\mathrm{x}, \mathrm{y})$ and the illumination veil I $(\mathrm{x}, \mathrm{y})$. Then we make the residual image $\mathrm{L}_{\mathrm{s}}(\mathrm{x}, \mathrm{y})$ divided by the illumination veil I (x,y), and get the final reflectance image $\rho(x, y)$ in Eq.(9). According to above inference, to estimate the illumination veil value must know the residual image $L_{s}(x, y)$. The residual image $L_{s}(x, y)$ estimation is relatively simple. we can subtract $\mathrm{V}(\mathrm{x}, \mathrm{y})$ from $\mathrm{L}(\mathrm{x}, \mathrm{y})$ to end up with residual image $\mathrm{L}_{\mathrm{s}}(\mathrm{x}, \mathrm{y})(\mathrm{Fig} .2$ (e) ). In Eq.(8), if $\rho(x, y) \rightarrow 1$, we get $L_{s}(x, y)=I(x, y)$. In other words, when all the light received by the object is reflected to the camera, the following Eq.(14 ) established:

$$
\mathrm{I}^{\mathrm{C}}(\mathrm{x}, \mathrm{y})=\lim _{\rho(\mathrm{x}, \mathrm{y}) \rightarrow 1} \mathrm{~L}_{\mathrm{S}}^{\mathrm{C}}(\mathrm{x}, \mathrm{y})
$$

As a matter of fact, for a small patch of color image[11], there must be at least one of $R, G$ or $B$ components that are close to the total reflection, whose reflectance coefficient approximately equals 1 . We put the residual image $L_{S}(x, y)$ through the non-linear filter, and we have:

$$
\mathrm{I}^{\mathrm{c}}(\mathrm{x}, \mathrm{y})=\max _{\mathrm{c} \in\{\mathrm{R}, \mathrm{G}, \mathrm{B}\}} \mathrm{L}_{\mathrm{S}}^{\mathrm{C}}(\mathrm{x}, \mathrm{y})
$$

Then we use the guided filter technology to optimize the image $I^{\mathrm{c}}(\mathrm{x}, \mathrm{y})($ Fig .2 (f) ). Here, the guidance image I is the image $\mathrm{I}^{\mathrm{C}}(\mathrm{x}, \mathrm{y})$ keeping the edge detail features. Then, we must choose the input image $\mathrm{p}$ by gray closing of dark channel and put them through guided filter. According to Eq.(9), we calculate the reflectance $\rho(\mathrm{x}, \mathrm{y})$. The reflectance image is the haze-free image after optimization. As shown in Fig .1(i), the contrast and brightness of the image dehazed is higher and more clear, comparing to the original image. 

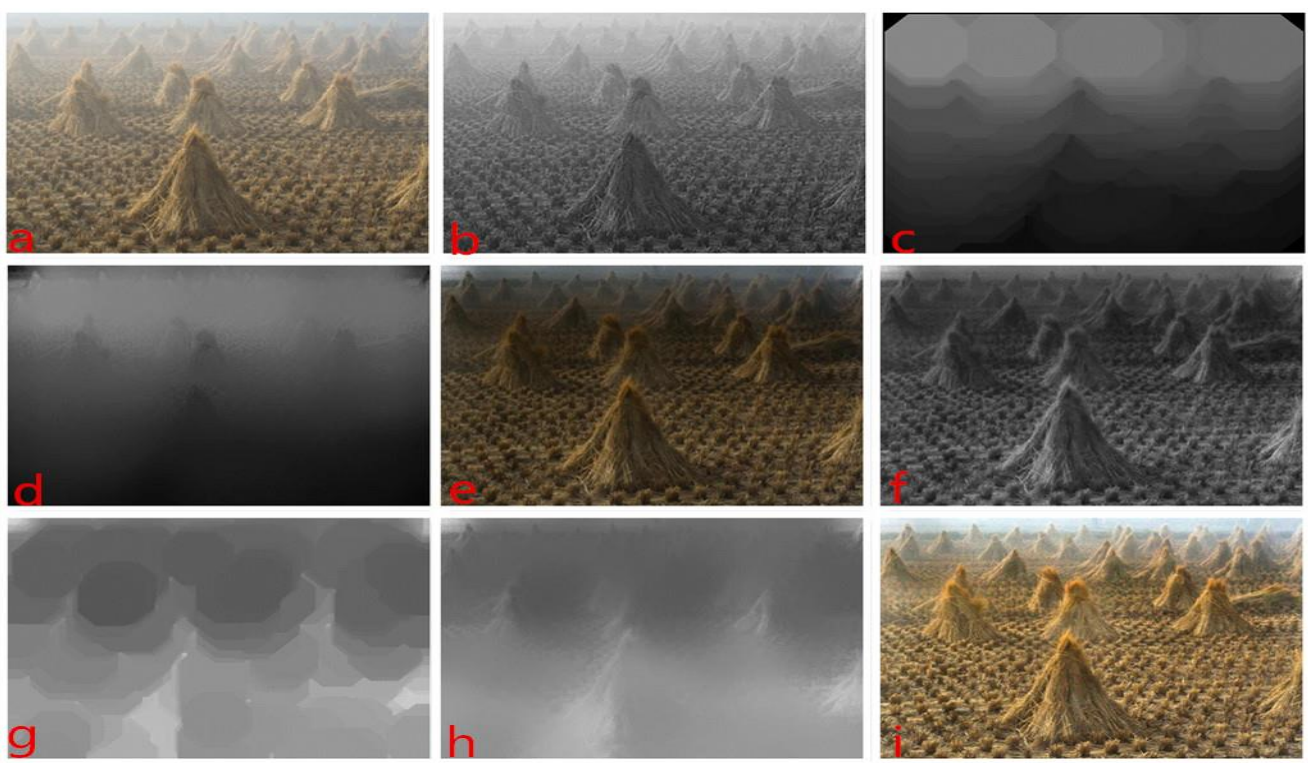

Figure 2. Restored image 'cones' by the proposed algorithm

(a) Hazy image (b) Dark channel of hazy image (c) Gray opening of dark channel (d) Atmosphere veil V(x,y) of the input image (e) Result of the residual image $L_{s}(x, y)$ (f) Max intensity of residual image (g)Gray closing of max intensity (h)Illumination veil $I(x, y)$ of the residual image $L_{s}(x, y)$ (i)Restored image $\rho(x, y)$ by the proposed method

\section{Scene reflectance Restoration}

As discussed above, the procedures of the proposed algorithm are given as follows:

1) Input the degraded color image $L(x, y)$ with $R G B$ channels.

2) According to $R, G$, and B channels at each pixel location, preliminarily estimate atmosphere veil $V_{d c}(x, y)$.

3) Refine $V(x, y)$ using guided filter on $V_{d c}(x, y)$ and $V^{\prime}(x, y)$.

4) Subtract $V(x, y)$ from $L(x, y)$ to end up with residual image $L_{s}(x, y)$.

5) According to $R, G$, and $B$ channels at each pixel location of $L_{s}(x, y)$, preliminarily estimate illumination veil $I(x, y)$.

6) Refine $I(x, y)$ using guided filter on $I^{c}(x, y)$ and $I^{\prime}(x, y)$.

7) Get $\rho(x, y)$ of the target using $\rho(x, y)=L_{s}(x, y) / I(x, y)$ as in Eq. (9) and truncate it to the region of [0 1].
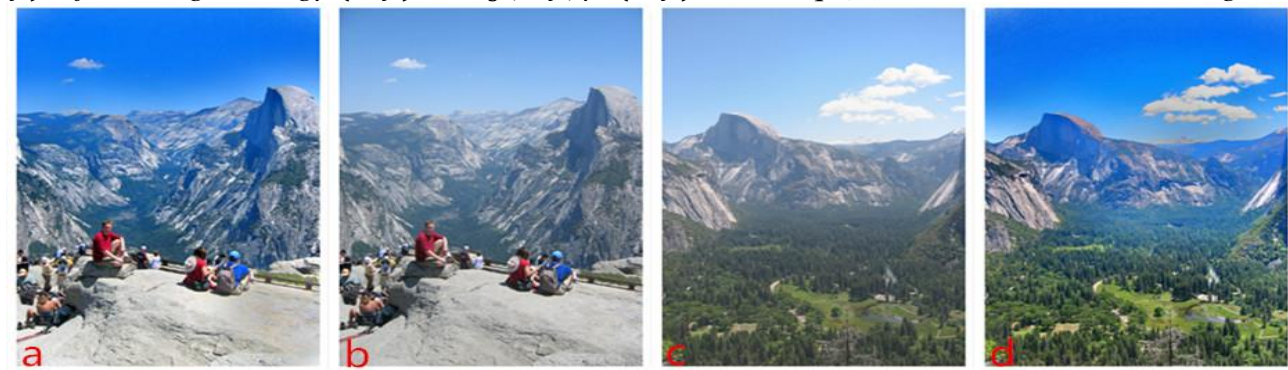

Figure 3. Comparable the results with the original image.

(b),(c) Hazy image; (a),(d) image restored. 

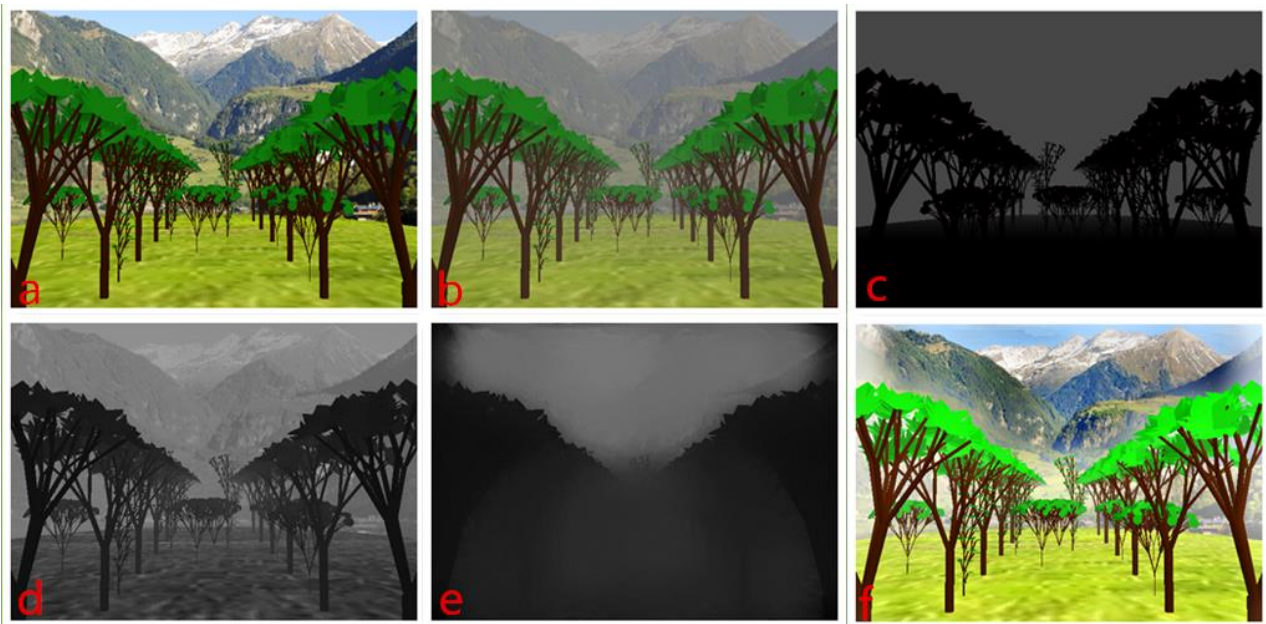

Figure 4. Comparable the results with the original image (we build a 3D simulation scene)

(a) Original image; (b) Foggy image ( $\mathrm{k}=0.03)$; (c) corresponding depth of hazy image; hazy image; (e) Dark channel optimized; (f ) Restored foggy image

\section{EXPERIMENTAL RESULTS AND COMPARISION}

The algorithm $\mathrm{He}$ proposed [4] estimated the atmospheric light roughly, used the dark channel to find a point at infinity and choose the value of atmospheric light of the point as a global constant. Due to the unknown depth of the scene, the point filtered is not necessarily the point of infinity through dark channel image. The algorithm this paper proposed combines the atmospheric light and the transmittance as an unknown quantity, avoids the risk of the atmospheric light A estimation and directly estimate the atmospheric veil $\mathrm{V}(\mathrm{x}, \mathrm{y})$, which increases the accuracy of the operation. Fig .5 shows that results are simple which are nature and clear through the algorithm proposed by He and this paper. Here compare the time of three image operation, experimental conditions for: Intel(R ) Core(TM)i5-4590 CPU @ 3.30GHz 8GB Memory, Matlab2010 Experiment platform, as shown in table 1

\section{OBJECTIVE EVALUATION}

In this paper, we use an approach proposed by Hautiere and etc [12] which consists in computing the ratio between the gradient of the visible edges between the image before and after contrast restoration The method defines the ratio e between the number of sets of new visible edges which have a local contrast above $5 \%$ of the original image and the restored image, as well as the average gradient ratio $(r)$ as the objective measurements of restored effect.

They are expressed as follows:

$$
\mathrm{e}=\frac{\mathrm{n}_{\mathrm{r}}-\mathrm{n}_{0}}{\mathrm{n}_{0}} \quad \overline{\mathrm{r}}=\frac{\overline{\mathrm{gr}_{\mathrm{r}}}}{\overline{\mathrm{g}_{0}}}
$$

Where $n_{0}$ and $n_{r}$ are the numbers of visible edges in the original image $\mathrm{L}(\mathrm{x}, \mathrm{y})$ and the restored image; $\overline{\mathrm{r}}, \overline{\mathrm{g}}$ is the average gradient of the original image and $\overline{g_{0}}$ denotes the average gradient of the restored image. As shown in table 1:

TABLE I . PRODUCED BY PROPOSED ALGORITHM AND COMPETING METHODS

\begin{tabular}{|c|c|c|c|c|c|c|c|c|c|}
\hline Image & & First line & & & econd lin & & & Third line & \\
\hline Algorithm & $\mathrm{t} / \mathrm{s}$ & $\mathrm{e}$ & $\overline{\mathrm{r}}$ & $\mathrm{t} / \mathrm{s}$ & $\mathrm{e}$ & $\overline{\mathrm{r}}$ & $\mathrm{t} / \mathrm{s}$ & $\mathrm{e}$ & 1.5924 \\
\hline $\mathrm{He}$ & 18.139 & 0.1380 & 1.3239 & 18.563 & 0.1253 & 1.3306 & 60.361 & 0.1647 & \\
\hline Trael & 4.323 & 0.1821 & 1.5134 & 4.396 & 0.2405 & 1.8953 & 14.174 & 0.1753 & 1.7451 \\
\hline Proposed & 1.475 & 0.1542 & 1.4260 & 1.557 & 0.1025 & 1.4954 & 3.516 & 0.1543 & 1.5849 \\
\hline
\end{tabular}



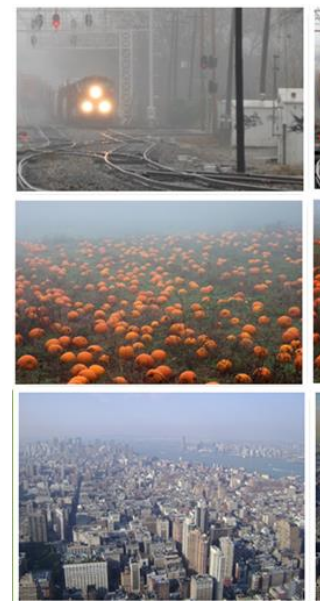

(a) Haze image
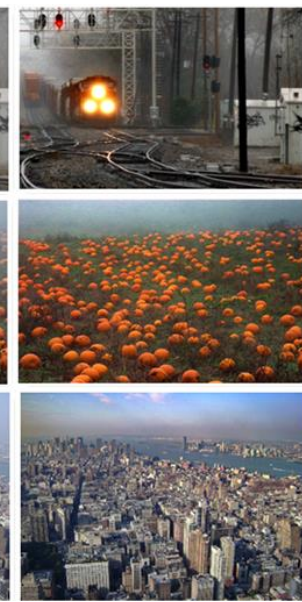

(b) He's result
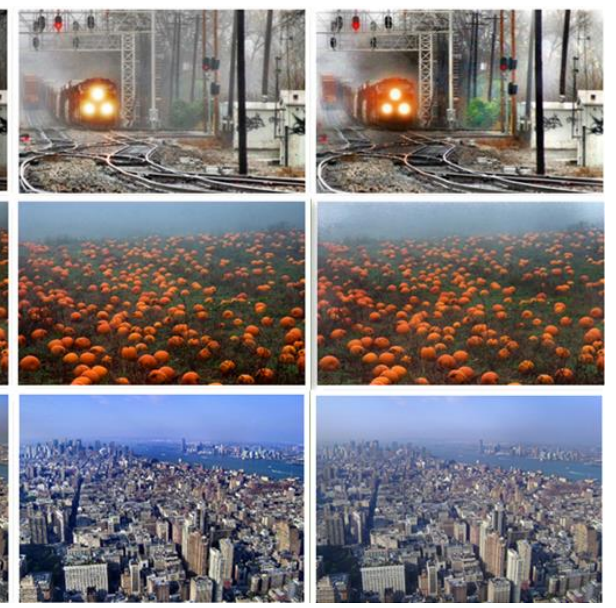

(c) Tarrel's result

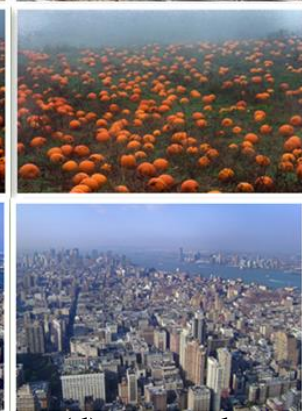

(d) our result

Figure 5. Comparison of different algorithm

\section{CONCLUSION}

With the rapid development of computer hardware and software and space science, image processing is more and more important. In the harsh environment, the quality of image directly affects the working efficiency of the computer vision system. The algorithm proposed avoids the risk of the atmospheric light A estimation which increases the accuracy of the operation. The main contribution of the paper is the development of a unified dehazing approach for hazy and un-illumination images. By the cross guided filter, a scene restoration scheme was proposed that produced good color rendition even for severe gray-world violations. This may reflect some underlying principles in the neural computations of consciousness, perhaps, even the visual representation of lightness and color.

\section{ACKNOWLEDGEMENTS}

This work was supported by Fundamental Research Funds for the Central Universities under Grant JB141307, National Nature Science Foundation of China (NSFC) under Grants 61201290, and other Grants 51205301, 61201089, 61305041, 61305040, the China Scholarship Council(CSC) and the National Institutes of Health Grants No. R01CA165255 of the United States.

\section{REFERENCES}

[1] Tan R. Visibility in bad weather from a single image. Proceedings of IEEE Conference on Computer Vision and Pattern Recognition. Washington, DC: IEEE Computer Society, 2008: 2347-2354.

[2] Fattal R. Single image dehazing[J]. ACM Transactions on Graphics, 2008, 27(3): 1-9.

[3] Tan K, Oakley P J. Physics-based approach to color image enhancement in poor visibility conditions [J]. Optical Society of America, 2001, 18(10): 2460-2467.

[4] He KaiMing,Sun Jian,Tang Xiaoou. Single image haze removal using dark channel prior[C]. In: Proceedings of the IEEE Conference on Computer Vision and Pattern Recognition Miami. USA: IEEE, 2009. 1956-1963.

[5] A. K. Tripathi and S. Mukhopadhyay, "Single Image Fog Removal Using Anisotropic Diffusion", IET Image processing, Volume 6, issue7, pp. 966 - 975, October 2012.

[6] Kim J H, Jang W D, Sim J Y, et al. Optimized contrast enhancement for real-time image and video dehazing [J]. Journal of Visual Communication and Image Representation, 2013.

[7] Xiao C, Gan J. Fast image dehazing using guided joint bilateral filter[J]. The Visual Computer, 2012, 28(6-8): 713-721.

[8] He, Kaiming, Jian Sun, and Xiaoou Tang. "Guided image filtering." Computer Vision-ECCV 2010. Springer Berlin Heidelberg, 2010. 1-14.

[9] Z. G. Li, J. H. Zheng, Z. J. Zhu, S. Q. Wu, W. Yao and S. Rahardja, "Content adaptive bilateral filtering," In IEEE 2013

International Conference on Multimedia and Expo, San Jose,

USA, Jul. 2013.

[10] C. Phan, S. Ha, and J. Jeon, "Adaptive guided image filtering for sharpness enhancement and noise removal," Advances in image and video technology, Springer Berlin Heidelberg, 2012.

[11] Sun, Wei, Long Han, Baolong Guo, Wenyan Jia, and Mingui Sun "A fast color image enhancement algorithm based on Max Intensity Channel." Journal of Modern Optics 61, no. 6 (2014): 466-477.

[12] Hautière, Nicolas, et al. Blind contrast enhancement assessment by gradient ratioing at visible edges. Image Analysis \& Stereology Journal 27.2 (2008): 87-95. 